\title{
Natural boundary value problems for weighted form Laplacians
}

\author{
Wojciech KozŁowski AND ANTONi PiERZCHALSKI
}

\begin{abstract}
The four natural boundary problems for the weighted form Laplacians $L=a d \delta+b \delta d, a, b>0$ acting on polynomial differential forms in the $n$-dimensional Euclidean ball are solved explicitly. Moreover, an algebraic algorithm for generating a solution from the boundary data is given in each case.
\end{abstract}

Mathematics Subject Classification (2000): 35J67 (primary); 35J25, 34K10 (secondary).

\section{Introduction}

Gradients in the sense of Stein and Weiss are $\mathrm{O}(n)$-irreducible parts of $\nabla$, the covariant derivative of an Riemannian manifold $M$ of dimension $n$ and of Riemannian metric $g$. For example, the bundle of differential p-forms is $\mathrm{O}(n)$-irreducible. But the target bundle of $\nabla$ acting $p$-forms splits. So, for any $p$-form $\omega$ we have the following decomposition ( $c f$. [26])

$$
\nabla \omega=\frac{1}{p+1} d \omega+\frac{1}{n-p+1} \operatorname{atr} \delta \omega+S \omega
$$

where atr is some operator of order zero described in [26]. As a result, we obtain three $\mathrm{O}(n)$-gradients: $d, \delta$ and $S$. The first two are the familiar exterior derivative and coderivative. The third operator $S$ completing the list, and defined just by the splitting, seems to be at least equally important. It is the only one of the three that has, like $\nabla$, an injective symbol. And that means the ellipticity. Roughly speaking, we can say that $S$ is carrying the ellipticity of $\nabla$. $S$ is called to be the Ahlfors operator.

In the particular case $p=1$, the operator $S$, being the symmetric and trace free part of $\nabla$, is one of the most important operators in conformal geometry: conformal Killing forms, or - by duality - vector fields, constitute its kernel. It is worth to notice that, in the case of $M=\mathbb{R}^{2}=\mathbb{C}$, the Ahlfors' operator becomes the CauchyRiemann one, so $S$ may be treated as its higher (even odd) dimensional extension. 
For more information on gradients see $[14,26]$ and, in particular, on the Ahlfors operator see [23, 24] and [25].

Recall that Ahlfors in [4] and [6] studied $S$ as an operator acting on vector fields $X$ in $\mathbb{R}^{n}$ :

$$
S X=\frac{1}{2}\left(D X+D X^{t}\right)-\frac{1}{n} \operatorname{trace}(D X) I,
$$

where $D X=\left(\partial X_{i} / \partial x_{j}\right), D X^{t}$ is the transpose of $D X$ and $I=\left(\delta_{i j}\right)$.

The adjoint operator is

$$
\left(S^{\star} \phi\right)_{i}=\sum_{j=1}^{n} \frac{\partial}{\partial x_{j}} \phi_{i j}
$$

So, the resulting differential operator $S^{\star} S$ maps vector fields into vector fields.

In the case of an arbitrary Riemannian manifold it is more convenient to replace vector fields by their duals: one forms. $S^{\star} S$ may be then written in its invariant shape [23]:

$$
S^{\star} S=\frac{n-1}{n} d \delta+\frac{1}{2} \delta d-\text { Ric },
$$

where Ric is the Ricci action on one-forms.

$S^{\star} S$ is strongly elliptic second order differential operator. In the case of Ricci flat manifold (and such is $M=\mathbb{R}^{n}$ ) it reduces to

$$
L_{a, b}=a d \delta+b \delta d
$$

where $a$ and $b$ are positive constants. The operators of this form will be called the weighted form Laplacains. These operators give a subclass of the class of so called non-minimal operators ( $c f$. [1] or [2]). Notice that the last formula enables getting an extension of the action of $L_{a, b}$ onto skew-symmetric forms of any degree $p$.

The extended operator $L_{a, b}$ is just the subject of our paper. It would seem that $L_{a, b}$ theory were just a version of that one for the Laplace-Beltrami operator $\Delta=\delta d+d \delta=L_{1,1}$. But, when $a \neq b$, this is not the case. In contrast to the situation which pertains for $\Delta$, the symbol of $L_{a, b}$, is no longer given by the metric tensor, so the situation is more subtle.

In the dimension three a version of $L_{a, b}$ acting on vector fields in a bounded domain was investigated in the context of an elastic body by H. Weyl [28]. In particular, the boundary problem under three different, physically motivated, boundary conditions were solved there. Ahlfors solved in [5] the Dirichlet boundary problem for $S^{\star} S$ in the $n$-dimensional hyperbolic ball. He used there the fact that the group of Möbius transformations of the unit ball (i.e., the group of isometries with respect to the hyperbolic metric) acts transitively. A Poisson type centre formula he derived there enabled therefore getting the value of a solution at any point of the ball. In the case of the Euclidean ball there is no such a tool. Yet, Reimann in [24] solved the Dirichlet problem for $S^{\star} S=0$ on vector fields in this case. In analogy to the 
classical procedure for the Dirichlet problem for the Laplace operator, consisting in expanding the functions on the sphere into a series of spherical harmonics, he decomposed the space of vector fields into some suitably chosen $\mathrm{O}(n)$-invariant subspaces Then he found a nice basis in each of the summands. Next Lipowski applied the Reimann method to solve the boundary problem under some other boundary conditions of Neumann type [19].

We are also going to adopt the Reimann's method here though his way of defining the Ahlfors operator of a higher order was passing to the space of tracefree symmetric tensors. Our way, in contrast to the Reimann one, is passing the space of skew-symmetric forms of an arbitrary degree $p$. The splitting onto $\mathrm{O}(n)$ invariant subspaces is then essentially different ([18]).

It seems to be interesting to find all the solutions for a complete list of some natural boundary conditions. Branson and the second named author observed in [8] that there is a general rule for generating a complete list of geometrically natural boundary conditions for $\mathrm{O}(n)$-gradients.

All conditions from the list are self-adjoint, and, in the case of elliptic gradients (cf. $[7,15,16])$, they constitute so called elliptic boundary conditions in the sense of Gilkey and Smith [12] (see also [10]).

Let us describe shortly that rule. For any gradient $G$ (one can think for a while that $\mathrm{G}$ is, e.g., $d, \delta$ or $S$ but the formula is really very general) we have ( $c f$. [21])

$$
\left(G^{*} G \omega_{1}, \omega_{2}\right)-\left(\omega_{1}, G^{*} G \omega_{2}\right)=\int_{\partial M}\left[g\left(\iota_{\nu} G \omega_{1}, \omega_{2}\right)-g\left(\omega_{1}, \iota_{\nu} G \omega_{2}\right)\right]
$$

where $\iota_{\nu} G \omega$ is the contraction of $G \omega$ with the unit vector $v$ normal to the boundary $\partial M$. To make $G^{*} G$ self-adjoint we have to accept boundary conditions annihilating the right hand side of (1.1), or stronger, annihilating each of the summands under the boundary integral. Now the unit normal $v$ will play its role. The original bundle we are dealing with (in our case the bundle of $p$-forms) is $\mathrm{O}(n)$-irreducible. But, it reduces at the boundary under the action of the subgroup $\mathrm{O}(n-1)$ of $\mathrm{O}(n)$ keeping $v$ invariant. As a result, by the Branching Rule, the original bundle splits at the boundary onto, say $s, \mathrm{O}(n-1)$-invariant subbundles. Denote by $\pi^{1}, \ldots, \pi^{s}$ the projections defined by the splitting. Then, by the orthogonality, $g\left(\omega_{1}, \iota_{\nu} G \omega_{2}\right)$ is equal to the sum

$$
g\left(\pi^{1} \omega_{1}, \pi^{1} \iota_{\nu} G \omega_{2}\right)+\ldots+g\left(\pi^{s} \omega_{1}, \pi^{s} \iota_{\nu} G \omega_{2}\right) .
$$

Now, there are $2^{s}$ candidates for elliptic boundary conditions, constructed as follows: For each $b=1, \ldots, s$, we choose exactly one of $\pi^{b} \omega_{1}$ and $\pi^{b} \iota_{\nu} G \omega_{2}$ and require it to vanish. For example, if we require to vanish the first multiplier in each summand we get the Dirichlet condition; if we require to vanish the other one we get the Neumann one. By other choices we get the whole their variety. The boundary conditions obtained that way seems to be in some sense "basic", at least from the point of view of the representation theory. Of course, we realize that the list may not contain some other geometrically or physically important conditions like Robin 
one etc. Of course, for different purposes or for some physical applications, we may always perturb by lower order operators. When we do so, we need to worry about losing the symmetry condition for the boundary integrand in (1.1), i.e., about loosing the self-adjointness. These perturbations will possibly take the form of order 0 operators, added either to the interior operator $G^{*} G$, or to the boundary operator $\omega \mapsto \iota_{\nu} G \omega$.

According to Branson and Pierzchalski [8], there are four such conditions on the list in the case of $\mathrm{O}(n)$-gradients acting on the space of differential forms of any degree on a Riemannian manifold $M$ with an nonempty boundary $\partial M$ :

Dirichlet boundary condition $(\mathcal{D})$ :

$$
\omega^{\mathrm{T}}=0 \text { and } \omega^{\mathrm{N}}=0 \text { on } \partial M .
$$

Absolute Boundary condition $(\mathcal{A})$ :

$$
\omega^{\mathrm{N}}=0 \text { and }(d \omega)^{\mathrm{N}}=0 \text { on } \partial M .
$$

Relative boundary condition $(\mathcal{R})$ :

$$
(\delta \omega)^{\mathrm{T}}=0 \text { and } \omega^{\mathrm{T}}=0 \text { on } \partial M .
$$

The fourth boundary condition $(\mathcal{B})$ :

$$
(\delta \omega)^{\mathrm{T}}=0 \text { and }(d \omega)^{\mathrm{N}}=0 \text { on } \partial M .
$$

Here $\omega^{\mathrm{T}}$ and $\omega^{\mathrm{N}}$ denote the tangent and the normal parts of $\omega$ at the boundary, respectively. The first three conditions are known to geometers. In particular, they appear in the Weyl's paper [28] mentioned above. The fourth one seems to be unknown. But, being natural, it should have a geometric or physical meaning.

Observe also a surprising symmetry with respect to the Hodge star operator $\star$. Namely, by the following known relations:

$$
\star \star= \pm 1, \quad(\star \omega)^{\mathrm{T}}= \pm \star\left(\omega^{\mathrm{N}}\right), \quad(\star \omega)^{\mathrm{N}}= \pm \star\left(\omega^{\mathrm{T}}\right)
$$

and

$$
\delta \omega= \pm \star d \star \omega, \quad d \omega= \pm \star \delta \star \omega
$$

it follows easily that the set of all the four boundary conditions $\{\mathcal{D}, \mathcal{A}, \mathcal{R}, \mathcal{B}\}$ is star-invariant. More precisely, each of the conditions $\mathcal{D}, \mathcal{B}$ is star-invariant, while the conditions $\mathcal{A}$ and $\mathcal{R}$ are star-symmetric each to the other.

In this paper we are going to solve all the four boundary problems $\mathcal{D}, \mathcal{A}, \mathcal{R}, \mathcal{B}$ for the operators $L_{a, b}$ acting onto differential forms of arbitrary degree $p$ in the Euclidean unit ball in $\mathbb{R}^{n}$. Excluding some exceptional cases namely: $p=n$ for the condition $\mathcal{R}, p=0$ for the condition $\mathcal{A}$ or $0<p<n$ for the condition $\mathcal{B}$ described in Proposition 4.6, in Proposition 4.8 or in Theorem 4.10, respectively, we 
prove the existence and uniqueness. In the exceptional cases we formulate necessary and sufficient conditions under which the existence also takes place. Moreover, we construct purely algebraic algorithm producing the solution explicitly from the polynomial boundary data in each case.

The condition $\mathcal{D}$ was the subject of the recent doctoral thesis of the first named author ([18]). This paper is a continuation.

ACKNOWLEDGEMENTS. The authors would like to express their gratitude to the referee for his valuable remarks that enabled essential improvements of the revised version.

\section{Preliminaries}

\subsection{Spherical harmonics - basic facts}

Suppose $n$ is non-negative integer, $n \geq 3$. If $x=\left(x^{1}, \ldots, x^{n}\right) \in \mathbb{R}^{n}$ and $m=$ $\left(m_{1}, \ldots, m_{n}\right), m_{j} \geq 0$, is a multi-index then $x^{m}=\left(x^{1}\right)^{m_{1}} \cdots\left(x^{n}\right)^{m_{n}},|m|=m_{1}+$ $\cdots+m_{n}$ and $m !=m_{1} ! \cdots m_{n}$ !. Put $\partial_{i}=\frac{\partial}{\partial x^{i}}, \partial_{i, j}^{2}=\partial_{i} \circ \partial_{j}$ and $D^{m}=\left(\partial_{1}\right)^{m_{1}} \circ$ $\cdots \circ\left(\partial_{n}\right)^{m_{n}}$. Denote by $\Sigma$ the unit sphere and by $B$ and $\bar{B}$ the open and the closed unit ball in $\mathbb{R}^{n}$, respectively.

Recall first the basic properties of homogeneous polynomials. For more details we refer to [9,27]. Let $\mathcal{P}_{k}$ denote the space of all homogeneous polynomials in $\mathbb{R}^{n}$ of degree $k$. For $f \in \mathcal{P}_{k}$ of form $f(x)=\sum_{|m|=k} a_{m} x^{m}$ define the differential operator associated with $f$ by $f(D)=\sum_{|m|=k} a_{m} D^{m}$. Obviously, $f(D)$ maps $\mathcal{P}_{l}$ into $\mathcal{P}_{l-k}$.

Define an inner product $(\cdot, \cdot)=(\cdot, \cdot)_{k}$ in $\mathcal{P}_{k}$ as follows; $(f, g)=f(D) g$, for $f, g \in \mathcal{P}_{k}$ It is worth to note that $(f, g)=\sum_{|m|=k} m ! a_{m} b_{m}$, where $f=$ $\sum_{|m|=k} a_{m} x^{m}$ and $g=\sum_{|m|=k} b_{m} x^{m}$. Clearly, for any $f \in \mathcal{P}_{k}, g \in \mathcal{P}_{l}$ and $h \in \mathcal{P}_{k+l},(g f, h)_{k+l}=(f, g(D) h)_{k}$. In particular, $\left(x^{j} f, h\right)=\left(f, \partial_{j} h\right)$. It means that the multiplication by $g$ and the operator $g(D)$ are formally adjoint each to the other.

For any $x \in \mathbb{R}^{n}$, denote by $|x|$ the Euclidean norm in $\mathbb{R}^{n}$. The polynomial $r^{2}$ defined by $r^{2}(x)=|x|^{2}$ is a member of $\mathcal{P}_{2}$. The differential operator $\Delta=$ $-r^{2}(D)=-\sum_{i=1}^{n} \partial_{i, i}^{2}$ is simply the classical Laplace operator on functions. Let $\mathcal{H}_{k}=\left\{h \in \mathcal{P}_{k}: \Delta h=0\right\}$ be the space of all harmonic homogeneous polynomials of degree $k$.

The classical fact on homogeneous polynomials is: If $f \in \mathcal{P}_{k}$ then there exist unique harmonic polynomials $h_{j} \in \mathcal{H}_{k-2 j}, 0 \leq j \leq l=[k / 2]$ such that

$$
f=h_{0}+r^{2} h_{1}+\cdots+r^{2 l} h_{l} \text {. }
$$

Put $f_{i}=h_{0}+r^{2} h_{1}+\cdots+r^{2 i} h_{i}, 0 \leq i \leq l$. Clearly, $f_{l}=f, f_{0}=h_{0}$ and for any 
$1 \leq i \leq l, f_{i-1}=f_{i}-r^{2 i} h_{i}$. We have

$$
h_{i}=\frac{1}{\gamma_{k-2 i, i}} \Delta^{i} f_{i}, \quad \text { for } i=0, \ldots, l,
$$

where

$$
\gamma_{k, m}= \begin{cases}1, & \text { if } m=0, \\ (-1)^{m} 2^{m} \prod_{j=0}^{m-1}(m-j)(n+2(k+m-j-1)), & \text { if } m>0 .\end{cases}
$$

Recall that spherical harmonics of degree $k$ are the restrictions of the polynomials from $\mathcal{H}_{k}$ to $\Sigma$. By the homogeneity, we may and we will, identify the space of the spherical harmonics of degree $k$ with $\mathcal{H}_{k}$.

The following identity will be an important tool in our farther considerations (for a very short and elegant proof we refer to [9]):

$$
\frac{1}{\operatorname{vol} \Sigma} \int_{\Sigma} f g \mathrm{~d} \Sigma=(n-2) \prod_{j=0}^{k} \frac{1}{2 j+n-2}(f, g)_{k}, \quad f, g \in \mathcal{H}_{k},
$$

where vol $\Sigma$ is the volume of $\Sigma$ and $\mathrm{d} \Sigma=\sum_{j=1}^{n}(-1)^{j-1} x^{j} d x^{1} \wedge \cdots \wedge d x^{j-1} \wedge$ $d x^{j+1} \wedge \cdots \wedge d x^{n}$ is the volume form of $\Sigma$.

Moreover, for any $f \in \mathcal{H}_{k}$ and $g \in \mathcal{H}_{l}, k \neq l$,

$$
\int_{\Sigma} f g \mathrm{~d} \Sigma=0
$$

\subsection{Homogeneous forms}

Consider any $p$-form $\omega$ defined in a subset $A \subset \mathbb{R}^{n}$. If $p=0$ we identify $\omega$ with a function on $A$. Assume additionally that any $p$-form, $p<0$, is the zero form. If $p \geq 1$ then $\omega$ has the unique expression

$$
\omega=\frac{1}{p !} \sum_{i_{1}, \ldots, i_{p}=1}^{n} \omega_{i_{1}, \ldots, i_{p}} d x^{i_{1}} \wedge \cdots \wedge d x^{i_{p}},
$$

where functions $\omega_{i_{1}, \ldots, i_{p}}: A \rightarrow \mathbb{R}$ called the coefficients, are skew-symmetric with respect to the indices.

Let $\Omega=\mathrm{dx}=d x^{1} \wedge \cdots \wedge d x^{n}$. be the volume form of $R^{n}$. Since any form $\omega$ of the maximal degree is proportional to $\Omega$, i.e., $\omega=f \Omega$, for some function $f$, we will sometimes identify $\omega$ with $f$.

If $\alpha$ and $\beta$ are $p$-forms defined in $A$, the pointwise inner product of $\alpha$ and $\beta$ is simply the function $\alpha \beta: A \rightarrow \mathbb{R}$ given by

$$
\alpha \beta=\frac{1}{p !} \sum_{i_{1}, \ldots, i_{p}=1}^{n} \alpha_{i_{1}, \ldots, i_{p}} \beta_{i_{1}, \ldots, i_{p}} .
$$

We will also write $\alpha^{2}$ instead of $\alpha \alpha$. 
Consider the vector field $v, v_{x}=x^{1} \partial_{1}+\cdots+x^{n} \partial_{n}$ and the 1-form $v^{\star}, v_{x}^{\star}=$ $x^{1} d x^{1}+\cdots+x^{n} d x^{n}$. Define two linear operators $\iota_{v}$ and $\varepsilon_{v}$ as follows. For any $p$-form $\omega$ let $\iota_{\nu} \omega=\omega(\nu, \cdot, \ldots, \cdot)$ if $p \geq 1$ and $\iota_{\nu} \omega=0$, if $p=0$, and let $\varepsilon_{v} \omega=v^{\star} \wedge \omega$. In particular, if $\omega$ is a 1 -form, $v^{\star} \omega=\iota_{\nu} \omega$. It is also known that $\iota_{v}$ is anti-derivation, i.e., for any $p$-form $\omega$ and any $q$-form $\eta$

$$
\iota_{v}(\eta \wedge \omega)=\left(\iota_{\nu} \eta\right) \wedge \omega+(-1)^{(-1) q} \eta \wedge \iota_{\nu} \omega .
$$

By the definitions of $\iota_{v}, \varepsilon_{v}$ and the obvious identity $v^{\star} v=r^{2}$, we can easily get that for any $\omega$,

$$
r^{2} \omega=\left(\iota_{\nu} \varepsilon_{v}+\varepsilon_{\nu} \iota_{v}\right) \omega
$$

Let $x_{0} \in \mathbb{R}^{n}$. We say that $\omega$ is tangential (respectively normal) at $x_{0}$ if $\left(\iota_{\nu} \omega\right)_{x_{0}}=0$ (respectively $\left.\left(\varepsilon_{\nu} \omega\right)_{x_{0}}=0\right)$. Clearly, each form is simultaneously tangential and normal at $0 \in \mathbb{R}^{n}$. Take now any subset $A \subset \mathbb{R}^{n}$. We say that $\omega$ is tangential (respectively normal) on $A$ if $\omega$ is tangential (respectively normal) at each point $z \in A$. Moreover, (2.6) implies that any (continuous) form being both tangential and normal must be trivial. The form $\omega$ may be uniquely expressed as a sum $\omega=$ $\omega^{\mathrm{T}}+\omega^{\mathrm{N}}$, where $\omega^{\mathrm{T}}$ and $\omega^{\mathrm{N}}$ are the tangential and the normal part of $\omega$, respectively. It is clear, that outside the origin,

$$
\begin{aligned}
\omega^{\mathrm{T}} & =\left(1 / r^{2}\right) \iota_{\nu} \varepsilon_{\nu} \omega, \\
\omega^{\mathrm{N}} & =\left(1 / r^{2}\right) \varepsilon_{\nu} \iota_{\nu} \omega .
\end{aligned}
$$

Define

$$
\begin{aligned}
& \pi^{\mathrm{T}} \omega=\omega^{\mathrm{T}}\left|\Sigma=\iota_{\nu} \varepsilon_{\nu} \omega\right| \Sigma, \\
& \pi^{\mathrm{N}} \omega=\omega^{\mathrm{N}}\left|\Sigma=\varepsilon_{\nu} \iota_{\nu} \omega\right| \Sigma,
\end{aligned}
$$

for any form $\omega$.

Let $d$ and $\delta$ denote (exterior) differential and codifferential operators, respectively. Relations between operators $d, \delta, \iota_{v}$ and $\varepsilon_{v}$ will play an important role in our considerations. The remaining part of this section is devoted to them.

As a consequence of the Green's formula we obtain that for any smooth forms $\omega$ and $\eta$ defined on the closure of $B$,

$$
\int_{B}(d \omega) \eta \mathrm{dx}=\int_{B} \omega(\delta \eta) \mathrm{dx}+\int_{\Sigma} \omega\left(\iota_{\nu} \eta\right) \mathrm{d} \Sigma .
$$

Let $\star$ denote Hodge star operator. Recall that for any $p$-form $\eta, \star \eta$ is the unique $(n-p)$-form such that for any $p$-form $\omega$,

$$
\omega \wedge \star \eta=(\omega \eta) \Omega .
$$

The Hodge star operator is an isometry, i.e., $(\star \omega)(\star \eta)=\omega \eta$ and it satisfies the identity $\star^{2}=\star \star=(-1)^{p(n-p)}$ on the space of $p$-forms. Moreover, one can easily check that for any $p$-form,

$$
(\star \omega)^{\mathrm{T}}=\star\left(\omega^{\mathrm{N}}\right), \quad(\star \omega)^{\mathrm{N}}=\star\left(\omega^{\mathrm{T}}\right) .
$$


In particular,

$$
\star \nu^{\star}=\iota_{\nu} \Omega, \quad \star \iota_{\nu} \Omega=(-1)^{n-1} \nu^{\star} .
$$

It is well known that

$$
\begin{aligned}
& \delta=(-1)^{n(p+1)+1} \star d \star, \\
& d=(-1)^{n(n-p) \star \delta \star,}
\end{aligned}
$$

on the space of differential $p$-forms.

In particular, for any differential $p$-form $\omega$,

$$
\begin{aligned}
& \star d \omega=(-1)^{p} \delta(\star \omega), \\
& \star \delta \omega=(-1)^{p+1} d(\star \omega) .
\end{aligned}
$$

Consequently,

$$
\begin{aligned}
& \star(d \omega)^{\mathrm{N}}=(-1)^{p}(\delta(\star \omega))^{\mathrm{T}}, \\
& \star(\delta \omega)^{\mathrm{T}}=(-1)^{p+1}(d(\star \omega))^{\mathrm{N}} .
\end{aligned}
$$

A $p$-form $\omega$ is called polynomial $p$-form if $\omega_{i_{1}, \ldots, i_{p}}$ 's are polynomials. Denote by $\Lambda^{p}$ the vector space of all polynomial $p$-forms in $\mathbb{R}^{n}$.

A polynomial $p$-form $\omega$ is called homogeneous if all its coefficients are polynomials from $\mathcal{P}_{k}$, for some $k$. Such a form will be also called $(p / k)$-form. Denote by $\Lambda_{k}^{p}$ the vector space of all $(p / k)$-forms. Manifestly, $\Lambda_{k}^{0}=\mathcal{P}^{k}$, and $\Lambda_{k}^{n}$ is, in a natural way, isomorphic to $\mathcal{P}^{k}$. Moreover, it is convenient to put $\Lambda_{k}^{p}=\{0\}$ if either $p<0$ or $k<0$. Now we may extend the inner product $(\cdot, \cdot)_{k}$ to the inner product $(\cdot \cdot \cdot)_{p, k}$ in the space $\Lambda_{k}^{p}$ as follows. For any $(p / k)$-forms $\omega$ and $\eta$ we put

$$
(\omega \mid \eta)_{p, k}=\frac{1}{p !} \sum_{i_{1}, \ldots, i_{p}=1}^{n}\left(\omega_{i_{1}, \ldots, i_{p}}, \eta_{i_{1}, \ldots, i_{p}}\right)_{k},
$$

where $\omega_{i_{1}, \ldots, i_{p}}$ 's and $\eta_{i_{1}, \ldots, i_{p}}$ 's denote coefficients of $\omega$ and $\eta$, respectively. Notice that $(\cdot \mid \cdot)_{0, k}$ and $(\cdot, \cdot)_{k}$ coincide. We will frequently write $(\cdot \mid \cdot)$ instead of $(\cdot \mid \cdot)_{p, k}$, if there is no ambiguity about the indices.

One can easily check that

$$
d \varepsilon_{v}=-\varepsilon_{v} d \quad \text { and } \quad \delta \iota_{v}=-\iota_{\nu} \delta .
$$

The following Proposition 2.1, Proposition 2.2 and Theorem 2.3 are proved in [18, Section 2.2].

Proposition 2.1. Suppose $\omega$ is a ( $p / k)$-form. We have the following identities

$$
\begin{aligned}
\delta \varepsilon_{\nu} \omega & =-\varepsilon_{\nu} \delta \omega-(n-p+k) \omega, \\
d \iota_{\nu} \omega & =-\iota_{\nu} d \omega+(p+k) \omega .
\end{aligned}
$$


Proposition 2.2. For any polynomial form $\omega$ we have

$$
\begin{aligned}
d\left(r^{2} \omega\right) & =r^{2} d \omega+2 \varepsilon_{\nu} \omega \\
\delta\left(r^{2} \omega\right) & =r^{2} \delta \omega-2 \iota_{\nu} \omega .
\end{aligned}
$$

Theorem 2.3. Let $d^{\star}$ and $\delta^{\star}$ denote the operators adjoint (with respect to the inner product $(\cdot, \cdot))$ to $d: \Lambda_{k}^{p} \rightarrow \Lambda_{k-1}^{p+1}$ and $\delta: \Lambda_{k}^{p} \rightarrow \Lambda_{k-1}^{p-1}$, respectively. Then, for any $(p / k)$-form $\omega$,

$$
\begin{aligned}
\delta^{\star} \omega & =-\varepsilon_{\nu} \omega, \\
d^{\star} \omega & =\iota_{\nu} \omega .
\end{aligned}
$$

\section{Weighted form Laplacians}

\subsection{Kernel of the weighted form Laplacian}

Let $a, b>0$. Consider the weighed form Laplacian $L=L_{a, b}=a d \delta+b \delta d$ In particular, if $a=b=1, L_{1,1}=d \delta+\delta d$ is just the Laplace-Beltrami operator. Notice that in the case of differential 0-forms, i.e., smooth functions, the LaplaceBeltrami operator $L_{1,1}$ and the classical Laplace operator coincide. Moreover, for any differential $p$-form $\omega$,

$$
(\Delta \omega)_{i_{1}, \ldots, i_{p}}=\Delta \omega_{i_{1}, \ldots, i_{p}} .
$$

Applying (2.10) and (2.11) we obtain that for any $a, b>0$

$$
\star L_{a, b}=L_{b, a},
$$

in analogy to the special case $a=b=1$.

From (3.1) it follows immediately that $\omega$ is harmonic, i.e., $\Delta \omega=0$, iff all its coefficients are harmonic. In particular, if $\omega$ is a polynomial form then each its coefficient is a harmonic polynomial, so $(p / k)$-form $\omega$ is harmonic if and only if each its coefficient $\omega_{i_{1}, \ldots, i_{p}}$ is in $\mathcal{H}_{k}$.

Denote by $\mathfrak{H}^{p}$ (respectively $\mathfrak{H}_{k}^{p}$ ) the space of all polynomial harmonic forms (respectively $(p / k)$-forms), i.e., $\mathfrak{H}^{p}=\operatorname{ker} \Delta \cap \Lambda^{p}$ and $\mathfrak{H}_{k}^{p}=\operatorname{ker} \Delta \cap \Lambda_{k}^{p}$. Consider $L=L_{a, b}$ as an operator $L: \Lambda_{k}^{p} \rightarrow \Lambda_{k-2}^{p}$, and let $\mathfrak{L}_{k}^{p}$ be its kernel, i.e., $\mathfrak{L}_{k}^{p}=$ ker $\Delta \cap \Lambda_{k}^{p}$. Clearly, if $k=0,1$ then $\Lambda_{k}^{p}=\mathfrak{L}_{k}^{p}=\mathfrak{H}_{k}^{p}$. Moreover, $\mathfrak{L}_{k}^{0}=\mathcal{H}_{k}$ and $\mathfrak{L}_{k}^{n}$ is in a natural way isomorphic to $\mathcal{H}_{k}$.

By (2.3), (2.4), (2.5) and (2.14) it follows that for any $\alpha \in \mathfrak{H}_{k}^{p}$ and $\beta \in \mathfrak{H}_{l}^{p}$

$$
\int_{\Sigma} \alpha \beta \mathrm{d} \Sigma= \begin{cases}s(k)(\alpha \mid \beta)_{p, k}, & \text { if } k=l, \\ 0, & \text { if } k \neq l,\end{cases}
$$


where

$$
s(k)=(\operatorname{vol} \Sigma)(n-2) \prod_{j=0}^{k} \frac{1}{2 j+n-2} .
$$

As a direct consequence of (2.1), (2.2) and (3.1) we obtain an algebraic algorithm of decomposing ( $p / k$ )-form into harmonic homogeneous forms. Namely, let $\omega \in \Lambda_{k}^{p}$ then there exist unique $\alpha_{j} \in \mathfrak{H}_{k-2 j}^{p}, 0 \leq j \leq l=[k / 2]$ such that

$$
\omega=\alpha_{0}+r^{2} \alpha_{1}+\cdots+r^{2 l} \alpha_{l} .
$$

Put $\omega_{i}=\alpha_{0}+r^{2} h_{1}+\cdots+r^{2 i} \alpha_{i}, 0 \leq i \leq l$. Clearly, $\omega_{l}=\omega, \omega_{0}=\alpha_{0}$ and for any $1 \leq i \leq l, \omega_{i-1}=\omega_{i}-r^{2 i} \alpha_{i}$. We have

$$
\alpha_{i}=\frac{1}{\gamma_{k-2 i, i}} \Delta^{i} \omega_{i}, \quad \text { for } i=0, \ldots, l,
$$

where

$$
\gamma_{k, m}= \begin{cases}1, & \text { if } m=0, \\ (-1)^{m} 2^{m} \prod_{j=0}^{m-1}(m-j)(n+2(k+m-j-1)), & \text { if } m>0 .\end{cases}
$$

By (3.4) we define the projection $\pi_{h}: \Lambda_{k}^{p} \rightarrow \mathfrak{H}^{p}$,

$$
\pi_{h}(\omega)=\alpha_{0}+\cdots+\alpha_{l} .
$$

Since any polynomial form has a unique decomposition onto a finite sum of homogeneous polynomial forms, we extend $\pi_{h}$ to the operator

$$
\pi_{h}: \Lambda^{p} \rightarrow \mathfrak{H}^{p} .
$$

The spaces

$$
\chi_{p, k}^{0}=\mathfrak{H}_{k}^{p} \cap \operatorname{ker} \delta \cap \operatorname{ker} \iota_{\nu}, \quad 0 \leq p \leq n, k \geq 0,
$$

will play a fundamental role in the decomposition of the ker $L$.

It is also convenient to put additionally $\chi_{q, l}^{0}=\{0\}$ if either $q<0$ or $l<0$. In [18] we proved

Proposition 3.1. Let $0 \leq p \leq n$ and $k \geq 0$. The space $\chi_{p, k}^{0}$ is trivial in each of the three cases

$$
\begin{gathered}
p>0, k=0, \\
p=n, \\
p=n-1, k=1 .
\end{gathered}
$$

Moreover, $\chi_{n-1,1}^{0}$ is a one-dimensional space spanned by $\iota_{v} \Omega$. 
Manifestly, $\mathfrak{H}_{k}^{0}=\chi_{0, k}^{0}=\mathcal{H}_{k}, \quad$ and $\quad \mathfrak{H}_{0}^{p}=\Lambda_{0}^{p}$. We need now to introduce some special linear operator:

$$
I_{L}(p . k)=\varepsilon_{v}-c_{L}(p, k) r^{2} d: \Lambda_{k}^{p} \rightarrow \Lambda_{k-1}^{p-1},
$$

where $c_{L}(p, k)$ is the constant;

$$
c_{L}(p, k)= \begin{cases}\frac{1}{2} \frac{2 b-(b-a)(n-p+k)}{a(p+k-2)+b(n-p+k-2)}, & \text { if } k \geq 2,0<p \leq n, \\ 0, & \text { otherwise. }\end{cases}
$$

Notice that our assumption ( $a, b>0$ and $n \geq 3$ ) ensure that $c_{L}(p, k)$ are welldefined. Observe also that in the special case $a=b=1$, i.e., if $L=\Delta$,

$$
c_{\Delta}(p, k)= \begin{cases}\frac{1}{n+2 k-4}, & \text { if } k \geq 2,0<p \leq n, \\ 0, & \text { otherwise. }\end{cases}
$$

We proved in [18, Section 3.3] that for any $0 \leq p \leq n$ and $k \geq 0$ the space $\mathfrak{L}_{k}^{p}$ is the direct sum of four mutually orthogonal subspaces:

$$
\mathfrak{L}_{k}^{p}=\chi_{p, k}^{0} \oplus^{\perp} d \chi_{p-1, k+1}^{0} \oplus^{\perp} \varepsilon_{\nu} d \chi_{p-2, k}^{0} \oplus^{\perp} I_{L}(p, k) \chi_{p-1, k-1}^{0} .
$$

And, in the special case of $\Delta$ :

$$
\mathfrak{H}_{k}^{p}=\chi_{p, k}^{0} \oplus^{\perp} d \chi_{p-1, k+1}^{0} \oplus^{\perp} \varepsilon_{\nu} d \chi_{p-2, k}^{0} \oplus^{\perp} I_{\Delta}(p, k) \chi_{p-1, k-1}^{0} .
$$

Moreover, $\chi_{p, k}^{0}, d \chi_{p-1, k+1}^{0}$ and $\varepsilon_{\nu} d \chi_{p-2, k}^{0}$ are subspaces of $\mathfrak{H}_{k}^{p}$. It is worth to say that some subspaces in (3.8) may degenerate sometimes. For example, when $p=1, \varepsilon_{\nu} d \chi_{p-2, k}=\{0\}$. Notice that the decomposition (3.8) is $\mathrm{SO}(n)$-invariant, but reducible in general. For more details see [18, Section 5.2].

Denote by $\mathbf{H}_{k}^{p}$ the space of all forms from $\Lambda_{k}^{p}$ which are both closed and coclosed, i.e., $\mathbf{H}_{k}^{p}=\left\{\omega \in \Lambda_{k}^{p}: d \omega=\delta \omega=0\right\}$. Clearly, $\mathbf{H}_{k}^{p} \subset \mathfrak{H}_{k}^{p}$, and by (3.9) we have the following

Corollary 3.2. For any $k \geq 0$ and $0 \leq p \leq n$ we have

$$
\mathbf{H}_{k}^{p}= \begin{cases}\mathbb{R}, & \text { if } p=k=0 \\ d \chi_{p-1, k+1}^{0}, & \text { otherwise. }\end{cases}
$$

Let us conclude the section with a relation that will be used in the proof of the uniqueness in the next section. Take differential $p$-forms $\varphi$ and $\psi$ defined on the closure of the unit ball $B$ and let $L=a d \delta+b \delta d$. Then by (2.7) we obtain

$$
\begin{aligned}
\int_{B}(L \varphi) \psi \mathrm{dx}= & a\left(\int_{B}(\delta \varphi)(\delta \psi) \mathrm{dx}+\int_{\Sigma}(\delta \varphi)\left(\iota_{\nu} \psi\right) \mathrm{d} \Sigma\right) \\
& +b\left(\int_{B}(d \varphi)(d \psi) \mathrm{dx}-\int_{\Sigma}\left(\iota_{\nu} d \varphi\right) \psi \mathrm{d} \Sigma\right) .
\end{aligned}
$$




\subsection{The action of $d$ and $\delta$ on $\mathfrak{L}_{k}^{p}$.}

This section has purely technical character. Proofs of Lemma 3.3-3.6 are left to the reader. To prove them it suffices to apply directly: Proposition 2.1, 2.2, Theorem 2.3 and Proposition 3.1.

Lemma 3.3. For any polynomial form $\omega$,

$$
\begin{aligned}
\Delta\left(\iota_{\nu} \omega\right) & =\iota_{\nu} \Delta \omega+2 \delta \omega, \\
\Delta\left(\varepsilon_{\nu} \omega\right) & =\varepsilon_{\nu} \Delta \omega-2 d \omega .
\end{aligned}
$$

\section{Lemma 3.4.}

(a) If $0<p \leq n$ and $k \geq 0$ then for any $\eta^{\prime}, \eta^{\prime \prime} \in \chi_{p-1, k+1}^{0}$,

$$
\left(d \eta^{\prime} \mid d \eta^{\prime \prime}\right)=(p+k)\left(\eta^{\prime} \mid \eta^{\prime \prime}\right) .
$$

In particular, $d: \chi_{p-1, k+1}^{0} \rightarrow \chi_{p, k}$ is one-to-one.

(b) If $2 \leq p \leq n$ and $k \geq 0$ then for any $\eta^{\prime}, \eta^{\prime \prime} \in \chi_{p-2, k}^{0}$,

$$
\left(\varepsilon_{\nu} d \eta^{\prime} \mid \varepsilon_{\nu} d \eta^{\prime \prime}\right)=(n-p+k)\left(d \eta^{\prime} \mid d \eta^{\prime \prime}\right) .
$$

In particular, $\varepsilon_{v}: d \chi_{p-2, k}^{0} \rightarrow \mathfrak{H}_{k}^{p}$ is one-to-one.

(c) If $p \geq 1$ and $k \geq 1$ then for any $\eta^{\prime}, \eta^{\prime \prime} \in \chi_{p-1, k-1}^{0}$,

$$
\left(I_{\Delta}(p, k) \eta^{\prime} \mid I_{\Delta}(p, k) \eta^{\prime \prime}\right)=\frac{(n+k-p-2)(n+2 k-2)}{n+2 k-4}\left(\eta^{\prime} \mid \eta^{\prime \prime}\right) .
$$

In particular, if $p \neq n$ or $k \neq 2$ then $I_{\Delta}(p, k): \chi_{p-1, k-1}^{0} \rightarrow \mathfrak{H}_{k}^{p}$ is one-toone.

Lemma 3.5. Suppose that $\omega \in \mathfrak{H}_{k}^{p}$ then we have

(i) If $\omega \in \chi_{p, k}^{0}$ then $\iota_{\nu} \omega=0$.

(ii) If $\omega \in d \chi_{p-1, k+1}^{0}, \omega=d \alpha$ then $\iota_{\nu} \omega=(p+k) \alpha$.

(iii) If $\omega \in \varepsilon_{v} d \chi_{p-2, k}^{0}, \omega=\varepsilon_{v} d \alpha$ then

$$
\iota_{\nu} \omega=\left(\frac{n+k-p}{n+2 k-2}\right) r^{2} d \alpha-(p+k-2) I_{\Delta}(p-1, k+1) \alpha .
$$

(iv) If $\omega \in I_{\Delta}(p, k) \chi_{p-1, k-1}^{0}, \omega=I_{\Delta}(p, k) \alpha$ then

$$
\iota_{\nu} \omega=r^{2} \alpha-c_{\Delta}(p, k)(p+k-2) \alpha .
$$




\section{Lemma 3.6.}

(i) For any $p, k, \chi_{p, k}^{0}$ is the space of tangential forms only. In particular, $\chi_{n, k}^{0}=$ $\{0\}$.

(ii) For any $p, k, \varepsilon_{v} d \chi_{p-2, k}^{0}$ is the space of normal forms only.

(iii) For any $\omega \in \chi_{p-1, k+1}^{0}$,

$$
\begin{aligned}
& (d \omega)^{\mathrm{T}}=d \omega-\frac{1}{r^{2}}(p+k) \varepsilon_{\nu} \omega, \\
& (d \omega)^{\mathrm{N}}=\frac{1}{r^{2}}(p+k) \varepsilon_{\nu} \omega .
\end{aligned}
$$

In particular on $\Sigma$,

$$
(d \omega)^{\mathrm{T}}=\left(\frac{n-p+k}{n+2 k}\right) d \omega-(p+k) I_{\Delta}(p, k+2) \omega .
$$

(iv) For any $\omega \in \chi_{p-1, k-1}^{0}$,

$$
\begin{aligned}
& \left(I_{L}(p, k) \omega\right)^{\mathrm{T}}=c_{L}(p, k)\left((p+k-2) \varepsilon_{v} \omega-r^{2} d \omega\right), \\
& \left(I_{L}(p, k) \omega\right)^{\mathrm{N}}=\left(1-c_{L}(p, k)(p+k-2)\right) \varepsilon_{v} \omega .
\end{aligned}
$$

(v) By (iii) and (iv) it follows that for any $\omega \in \chi_{p-1, k-1}^{0}$,

$$
\begin{array}{ll}
\left(I_{L}(p, k) \omega\right)^{\mathrm{T}} & =-c_{L}(p, k) \frac{1}{r^{2}}(d \omega)^{\mathrm{T}}, \\
\left(I_{L}(p, k) \omega\right)^{\mathrm{N}} & = \begin{cases}\varepsilon_{\nu} \omega & \text { if } p=k=1, \\
\left(\frac{1-c_{L}(p, k)(p+k-2)}{p+k-2}\right) r^{2}(d \omega)^{\mathrm{N}} & \text { otherwise. }\end{cases}
\end{array}
$$

(vi) For any $\omega \in \chi_{p-2, k}^{0}$,

$$
\delta \varepsilon_{\nu} d \omega=-(n-p+k) d \omega .
$$

So,

$$
\begin{aligned}
\left(\delta \varepsilon_{\nu} d \omega\right)^{\mathrm{T}} & =-(n-p+k)(d \omega)^{\mathrm{T}}, \\
\left(\delta \varepsilon_{v} d \omega\right)^{\mathrm{N}} & =-(n-p+k)(d \omega)^{\mathrm{N}} .
\end{aligned}
$$

(vii) For any $\omega \in \chi_{p-1, k-1}^{0}$,

$$
\delta I_{L}(p, k) \omega=\left(2 c_{L}(p, k)(p+k-2)-(n-p+k)\right) \omega .
$$

Thus $\delta I_{L}(p, k) \chi_{p-1, k-1}^{0}$ consists of tangential forms only. 
(viii) For any $\omega \in \chi_{p-1, k-1}^{0}$,

$$
d I_{L}(p, k) \omega=-\left(1+2 c_{L}(p, k)\right) \varepsilon_{v} d \omega .
$$

Thus $d I_{L}(p, k) \chi_{p-1, k-1}^{0}$ consists of normal forms only.

Let $u_{L}(p, k)=\left(2 c_{L}(p, k)(p+k-2)-(n-p+k)\right)$, the right hand side of (vii). We have

$$
u_{L}(p, k)= \begin{cases}-\frac{b(k+n-p-2)(2 k+n-2)}{a(k+p-2)+b(k+n-p-2)}, & \text { if } k \geq 2,0<p \leq n \\ -(n-p+k), & \text { otherwise. }\end{cases}
$$

The following property will be important in the next section:

$$
u_{L}(p, k)=0 \text { iff }(p=n \text { and } k=2) \text { or }(p=n \text { and } k=0) .
$$

\subsection{Projection formulae}

Fix $\alpha \in \mathfrak{L}_{k}^{p}$. By (3.8) there exist unique $\alpha^{1} \in \chi_{p, k}^{0}, \alpha^{2} \in d \chi_{p-1, k+1}^{0}, \alpha^{3} \in$ $\varepsilon_{v} d \chi_{p-2, k}^{0}$ and $\alpha^{4} \in I_{L}(p, k) \chi_{p-1, k-1}^{0}$ such that

$$
\alpha=\alpha^{1}+\alpha^{2}+\alpha^{3}+\alpha^{4} .
$$

For each $1 \leq i \leq 4$ define the projection $\pi^{i}=\pi_{k}^{i}$ by $\pi^{i}(\alpha)=\alpha^{i}$. Let $\eta^{2} \in$ $\chi_{p-1, k+1}^{0}, \eta^{3} \in \chi_{p-2, k}^{0}$ and $\eta^{4} \in \chi_{p-1, k-1}^{0}$ be such that $\alpha^{2}=d \eta^{2}, \alpha^{3}=\varepsilon_{v} d \eta^{3}$ and $\alpha^{4}=I_{L}(p, k) \eta^{4}$. It can be shown that if $\alpha^{i} \neq 0$ then the corresponding $\eta^{i}$ is uniquely determined. If $\alpha^{i}=0$ then we put $\eta^{i}=0$. Define the maps $\sigma^{i}=\sigma_{k}^{i}$, $i=2,3,4$, by $\sigma^{i}(\alpha)=\eta^{i}$. Let $\mathbf{j}$ denote the identity map on $\mathfrak{L}_{k}^{p}$. Clearly, we have

$$
\pi^{1}=\mathbf{j}-\pi^{2}-\pi^{3}-\pi^{4}, \quad \pi^{2}=d \sigma^{2}, \quad \pi^{3}=\varepsilon_{\nu} d \sigma^{3}, \quad \pi^{4}=I_{L}(p, k) \sigma^{4} .
$$

The maps $\sigma^{i}, i=2,3,4$, can be expressed by (cf. [18, Section 4.3])

$$
\begin{aligned}
\sigma^{2} & = \begin{cases}0, & \text { if } p=0, \\
\frac{1}{p+k} \iota_{\nu}\left(\mathbf{j}-\pi^{4}-\pi^{3}\right), & \text { otherwise. }\end{cases} \\
\sigma^{3} & = \begin{cases}0, & \text { if } k=0 \text { or } p=0,1, \\
-\frac{1}{(n-p+k)(p+k-2)} \iota_{\nu} \delta, & \text { otherwise. }\end{cases} \\
\sigma^{4} & = \begin{cases}0, & \text { if } k=0 \text { or } p=0, \\
\frac{1}{2 c_{L}(p+k-2)-(n-p+k)}\left(\delta+(n-p+k) d \sigma^{3}\right), & \text { otherwise. }\end{cases}
\end{aligned}
$$




\section{Four natural boundary conditions}

In the whole section we will assume that the weighted form Laplacian $L=L_{a, b}$ is fixed.

The image of $\Lambda^{p}$ under $\pi^{\mathrm{T}}$ (respectively $\pi^{\mathrm{N}}$ ) will be denoted by $\mathcal{T}^{p}$ (respectively $\mathcal{N}^{p}$ ), i.e.,

$$
\begin{aligned}
\mathcal{T}^{p} & =\pi^{\mathrm{T}}\left(\Lambda^{p}\right), \\
\mathcal{N}^{p} & =\pi^{\mathrm{N}}\left(\Lambda^{p}\right) .
\end{aligned}
$$

\subsection{Dirichlet condition $\mathcal{D}$}

Theorem 4.1 (Dirichlet boundary condition). For any $\omega \in \mathcal{T}^{p}$ and $\eta \in \mathcal{N}^{p}$, there exists a unique $\varphi \in \Lambda^{p}$ such that $L \varphi=0$ in $B$ with

$$
\varphi^{\mathrm{T}}=\omega \quad \varphi^{\mathrm{N}}=\eta \quad \text { on } \Sigma .
$$

A purely algebraic proof of Theorem 4.1 may be found in [18, Section 4.2]. Also an algorithm for a solution is constructed there. By the reason of the symmetry of the four boundary problems let us recall it shortly here again.

Take $\tilde{\omega}, \tilde{\eta} \in \Lambda^{p}$ such that $\pi^{\mathrm{T}} \tilde{\omega}=\omega$ and $\pi^{\mathrm{N}} \tilde{\eta}=\eta$. Put $\alpha=\iota_{\nu} \varepsilon_{\nu} \tilde{\omega}+\varepsilon_{\nu} \iota_{\nu} \tilde{\eta}$. Then we have

$$
\pi^{\mathrm{T}} \alpha=\omega, \quad \pi^{\mathrm{N}} \alpha=\eta
$$

Suppose that $\alpha$ has the following decomposition

$$
\alpha=\alpha_{0}+r^{2} \alpha_{1}+\cdots+r^{2 l} \alpha_{l},
$$

where $\alpha_{j} \in \mathfrak{H}_{j}^{p}$. (To obtain (4.2) decompose polynomial form into homogeneous component and then apply the procedure described in (3.4) and (3.5).) Let $\eta_{j}=$ $\sigma_{j}^{4} \alpha_{j}$. Put

and

$$
\alpha_{j}^{\prime}=\alpha_{j}+\left(1-r^{2}\right)\left(c_{L}(p, k)-c_{\Delta}(p, k)\right) d \eta_{j},
$$

$$
\varphi=\sum_{j=0}^{l} \alpha_{j}^{\prime} .
$$

Then $L \varphi=0$. Moreover, $\alpha|\Sigma=\varphi| \Sigma$. Thus a polynomial form $\varphi$ is a solution to our equation, by (4.1).

To prove the uniqueness suppose that a $p$-form $\varphi$ is a solution to the problem $L \varphi=0$ with $\varphi^{\mathrm{T}}=0$ and $\varphi^{\mathrm{N}}=0$ on $\Sigma$. We have $\iota_{\nu} \varphi=\iota_{\nu}\left(\varphi^{\mathrm{N}}\right)=0$ on $\Sigma$, and $\left(\iota_{\nu} d \varphi\right) \varphi=\left(\iota_{\nu} d \varphi\right) \varphi^{T}=0$ on $\Sigma$. Consequently, applying (3.10) to $\psi=\varphi$ we have

$$
0=a \int_{B}(\delta \varphi)^{2} \mathrm{dx}+b \int_{B}(d \varphi)^{2} \mathrm{dx} .
$$

Thus $\varphi$ is both closed and co-closed, so it is harmonic. By (3.1), each coefficient of $\varphi$ is a harmonic function in $B$ that trivializes on $\Sigma$. The assertion follows now by the ordinary Maximum Principle for harmonic functions. 


\subsection{Absolute $\mathcal{A}$ and relative $\mathcal{R}$ boundary condition}

Theorem 4.2 (Relative boundary condition). Let $0 \leq p<n$. For any $\omega \in \mathcal{T}^{p-1}$ and $\eta \in \mathcal{T}^{p}$ there exists a unique $\varphi \in \Lambda^{p}$ such that $L \varphi=0$ in $B$ with

$$
(\delta \varphi)^{\mathrm{T}}=\omega \quad \varphi^{\mathrm{T}}=\eta \quad \text { on } \Sigma .
$$

Theorem 4.3 (Absolute boundary condition). Let $0<p \leq n$. For any $\omega \in$ $\mathcal{N}^{p+1}$ and $\eta \in \mathcal{N}^{p}$ there exists a unique $\varphi \in \Lambda^{p}$ such that $L \varphi=0$ and

$$
(d \varphi)^{\mathrm{N}}=\omega \quad \varphi^{\mathrm{N}}=\eta \quad \text { on } \Sigma .
$$

As it was mentioned it the Introduction the relative $\mathcal{R}$ and absolute $\mathcal{A}$ boundary conditions are star-symmetric each to the other. In fact, by (2.8), (2.12)-(2.13) and (3.2) we have the following

Proposition 4.4. Let $\omega$ and $\eta$ be a continuous tangential ( $p-1)$-form and $p$-form, respectively, defined on $\Sigma$. A p-form $\varphi$ is a solution to the relative boundary condition $L_{a, b} \varphi=0$ in $B$ with $(\delta \varphi)^{\mathrm{T}}=\omega$ and $\varphi^{\mathrm{T}}=\eta$ on $\Sigma$ iff $(n-p)$-form $\star \varphi$ is a solution to absolute boundary condition $L_{b, a} \varphi=0$ in $B$ with $(d \varphi)^{\mathrm{N}}=(-1)^{p+1} \star \omega$ and $\varphi^{\mathrm{N}}=\star \eta$ on $\Sigma$.

Proof of Theorem 4.2 (Existence and an algorithm). Take polynomial forms $\tilde{\omega}$ and $\tilde{\eta}$ such that $\omega=\pi^{\mathrm{T}} \tilde{\omega}$ and $\eta=\pi^{\mathrm{T}} \tilde{\eta}$.

Assume first that $\tilde{\eta}=\tilde{\eta}_{k} \in \mathfrak{H}_{k}^{p}$ and $\tilde{\omega}=\tilde{\omega}_{l} \in \mathfrak{H}_{l}^{p-1}$. If $p=0$ then the relative boundary condition reduces to the Dirichlet one, so we may assume that $p>0$.

By (3.9) we get the decompositions:

$$
\begin{aligned}
& \tilde{\eta}_{k}=\alpha^{\prime}+d \beta^{\prime}+\varepsilon_{\nu} d \tau^{\prime}+I_{\Delta}(p, k) \theta^{\prime} \\
& \tilde{\omega}_{l}=\alpha+d \beta+\varepsilon_{\nu} d \tau+I_{\Delta}(p-1, l) \theta
\end{aligned}
$$

where

$$
\alpha^{\prime} \in \chi_{p, k}^{0}, \quad \beta^{\prime} \in \chi_{p-1, k+1}^{0}, \quad \tau^{\prime} \in \chi_{p-2, k}^{0}, \quad \theta^{\prime} \in \chi_{p-1, k-1}^{0},
$$

and

$$
\alpha \in \chi_{p-1, l}^{0}, \quad \beta \in \chi_{p-2, l+1}^{0}, \quad \tau \in \chi_{p-3, l}^{0}, \quad \theta \in \chi_{p-2, l-1}^{0} .
$$

Put

$$
\psi_{k}=\alpha^{\prime}+d \beta^{\prime}-c_{\Delta}(p, k) d \theta^{\prime} .
$$

Clearly, $L \psi_{k}=0, \delta \psi_{k}=0$ and on $\Sigma, \psi_{k}^{\mathrm{T}}=\tilde{\eta}^{\mathrm{T}}=\eta$ by (i), (ii) and (v) of Lemma 3.6.

By (3.11), it follows, under our assumption $(0<p<n)$, that $u_{L}(p, l+1) \neq 0$ and $n-p+l+1 \neq 0$. Put

$$
\begin{aligned}
\zeta_{l}= & \frac{1}{u_{L}(p, l+1)}\left(I_{L}(p, l+1) \alpha+c_{L}(p, l+1) d \alpha\right) \\
& -\frac{1}{n-p+l+1} \varepsilon_{\nu} d \beta \\
& +\frac{1}{n-p+l-1} c_{\Delta}(p-1, l) \varepsilon_{\nu} d \theta .
\end{aligned}
$$


Notice that the constant $n-p+l-1$ in the denominator of (4.6) is equal to 0 iff $p=n-1$ and $l=0$. But then $\theta=0$, so $\varepsilon_{v} d \theta=0$ and the last term in the above sum disappears in this case.

One can easily check that $L \zeta_{l}=0, \zeta_{l}^{\mathrm{T}}=0$ and on $\Sigma,\left(\delta \zeta_{l}\right)^{\mathrm{T}}=\tilde{\omega}^{\mathrm{T}}=\omega$ by Proposition 2.2, (ii) and (v) and (vi) of Lemma 3.6.

Finally, our solution is of form

$$
\varphi=\varphi_{k l}=\eta_{k}+\zeta_{l}
$$

In the general case we proceed as follows. There exist $m \geq 0$ such that $\tilde{\omega} \mid \Sigma=$ $\sum_{j=0}^{m} \tilde{\omega}_{j} \mid \Sigma$ and $\tilde{\eta}\left|\Sigma=\sum_{j=0}^{m} \tilde{\eta}_{j}\right| \Sigma$ with $\tilde{\omega}_{j}, \tilde{\eta}_{j} \in \mathfrak{H}_{j}^{p}$. (Some of the forms in the above decomposition may equal zero). Then,

$$
\varphi=\sum_{j=0}^{m} \varphi_{i i}
$$

is a solution to our equation.

Uniqueness of solutions. Suppose that a polynomial $p$-form $\varphi$ is a solution to $L \varphi=$ 0 in $B, \varphi^{\mathrm{T}}=0$ and $(\delta \varphi)^{\mathrm{T}}=0$. Then $(\delta \varphi)\left(\iota_{\nu} \varphi\right)=(\delta \varphi)^{\mathrm{T}}\left(\iota_{\nu} \varphi\right)=0$ on $\Sigma$ and $\left(\iota_{\nu} d \varphi\right) \varphi=\left(\iota_{\nu} d \varphi\right) \varphi^{\mathrm{T}}=0$ on $\Sigma$. Consequently (4.3) holds. Thus the polynomial $p$-form $\varphi$ is both closed and co-closed, so $\varphi=\sum_{j=0}^{m} \varphi_{j}$, with $\varphi_{j} \in \mathbf{H}_{j}^{p}$. Since, $p>0$, by Corollary 3.2 it follows that $\varphi_{j}=d \omega_{j}$ where $\omega_{j} \in \chi_{p-1, j+1}^{0}$. Applying Lemma 3.6 (iii) we obtain that on $\Sigma$

$$
0=\sum_{j=0}^{m}\left(d \omega_{j}\right)^{\mathrm{T}}=\sum_{j=0}^{m}\left(\frac{n-p+j}{n+2 j} d \omega_{j}-(p+j) I_{\Delta}(p, j+2) \omega_{j}\right) .
$$

Denote the last sum (not its restriction to $\Sigma$ ) by $\alpha$. Since $\alpha$ is a harmonic polynomial form and is equal to zero on $\Sigma, \alpha$ must be zero, by uniqueness of solution to the Dirichlet condition. Therefore, by (3.9) for each $0 \leq j \leq m$,

$$
\frac{n-p+j}{n+2 j} d \omega_{j}=0 \quad \text { and } \quad(p+j) I_{\Delta}(p, j+2) \omega_{j}=0 .
$$

Since $p<n$ and $j \geq 0,(n-p+j) /(n+2 j) \neq 0$. Thus $d \omega_{j}=0$, so $\omega_{j}=0$ by (a) of Lemma 3.4. Consequently, $\varphi_{j}=0$, so we have the uniqueness.

Algorithm to Theorem 4.3. Take polynomial forms $\tilde{\omega}$ and $\tilde{\eta}$ such that $\omega=\pi^{\mathrm{T}} \tilde{\omega}$ and $\eta=\pi^{\mathrm{T}} \tilde{\eta}$. Observe that if $p=n$ then the absolute boundary condition reduces to the Dirichlet one. So we may assume that $p<n$.

Suppose first that $\tilde{\eta}=\tilde{\eta}_{k} \in \mathfrak{H}_{k}^{p}$ and $\tilde{\omega}=\tilde{\omega}_{l} \in \mathfrak{H}_{l}^{p+1}$.

By (3.9) we get the decompositions:

$$
\begin{aligned}
& \tilde{\eta}_{k}=\alpha^{\prime}+d \beta^{\prime}+\varepsilon_{\nu} d \tau^{\prime}+I_{\Delta}(p, k) \theta^{\prime} \\
& \tilde{\omega}_{l}=\alpha+d \beta+\varepsilon_{\nu} d \tau+I_{\Delta}(p+1, l) \theta
\end{aligned}
$$


where

$$
\alpha^{\prime} \in \chi_{p, k}^{0}, \quad \beta^{\prime} \in \chi_{p-1, k+1}^{0}, \quad \tau^{\prime} \in \chi_{p-2, k}^{0}, \quad \theta^{\prime} \in \chi_{p-1, k-1}^{0},
$$

and

Put

$$
\alpha \in \chi_{p+1, l}^{0}, \quad \beta \in \chi_{p, l+1}^{0}, \quad \tau \in \chi_{p-1, l}^{0}, \quad \theta \in \chi_{p, l-1}^{0} .
$$

$$
\psi_{k}=d \beta^{\prime}+\varepsilon_{\nu} d \tau^{\prime}+\left(\frac{1-c_{\Delta}(p, k)(p+k-2)}{p+k-2}\right) d \theta^{\prime} .
$$

Notice that if the denominator $p+k-2$ equals zero then $d \theta^{\prime}=0$. Thus, in this case, we omit the last term in the sum above.

One can check that $L \psi_{k}=0$ and $d \psi_{k}=0$. Moreover, by (i), (ii) and (v) of Lemma 3.6 we conclude that on $\Sigma, \psi_{k}^{\mathrm{N}}=\eta$.

Define $\zeta_{l}$ as follows: If $p=1$ and $l=0$ then

$$
\zeta_{l}=\beta,
$$

otherwise

$$
\begin{aligned}
\zeta_{l}= & \beta+\left(\frac{1-c_{L}(p, l+1)(p+l-1)}{\left(1+2 c_{L}(p, l+1)\right)(p+l-1)}\right) d \tau \\
& -\frac{1}{1+2 c_{L}(p, l+1)} I_{L}(p, l+1) \tau \\
& +\left(\frac{1-c_{\Delta}(p+1, l)(p+l-1)}{p+l-1}\right) \theta .
\end{aligned}
$$

Notice that $1+2 c_{L}(p, l+1) \neq 0$. Moreover, if $p+l-1=0$ then both $d \tau$ and $\theta$ are zero, so in this case, we cancel the third and the fourth terms in the sum above.

Now, one can check that $L \zeta_{l}=0$ and, by Lemma 3.6, we have $\left(d \zeta_{l}\right)^{\mathrm{N}}=\omega$ and $\zeta_{l}^{\mathrm{N}}=0$ on $\Sigma$. Consequently, $\varphi=\varphi_{k l}=\psi_{k}+\zeta_{l}$ is a solution to our equation.

In the general case we repeat the last part of the algorithm from Theorem 4.2.

One may wonder why we exclude the case $p=n$ from Theorem 4.2. Since any $n$-form is normal in the case of $p=n$ we drop out the condition $\varphi^{T}=\eta$ on $\Sigma$. It turns out that in this case, a solution may not exist. Moreover if it exists, it is not unique. Namely, we have the following

Lemma 4.5. Let $\omega$ be a continuous tangential $(n-1)$-form defined on $\Sigma$. If a $n$-form $\varphi$ (smooth on the closure of $B$ ) is a solution to the equation $L \varphi=0$ in $B$ with

$$
(\delta \varphi)^{\mathrm{T}}=\omega \quad \text { on } \Sigma,
$$

then

$$
\int_{\Sigma} \omega\left(\iota_{v} \Omega\right) \mathrm{d} \Sigma=0
$$

Any other solution to this equation is of the form $\varphi+\operatorname{const} \Omega$. 
Proof. Since $\varphi$ is a $n$-form, $d \varphi=0$. Next, on $\Sigma$ we have

$$
(\delta \varphi)\left(\iota_{\nu} \Omega\right)=(\delta \varphi)^{\mathrm{T}}\left(\iota_{\nu} \Omega\right)=\omega\left(\iota_{\nu} \Omega\right) .
$$

Using now (3.10) for $\psi=\Omega$, we obtain

$$
0=a\left(\int_{B}(\delta \varphi)(\delta \Omega) \mathrm{dx}+\int_{\Sigma}(\delta \varphi)\left(\iota_{\nu} \Omega\right) \mathrm{d} \Sigma\right)=\int_{\Sigma} \omega\left(\iota_{\nu} \Omega\right) \mathrm{d} \Sigma,
$$

for $\Omega$ is co-closed.

To prove the second statement of the theorem it suffices to show that if $\varphi$ is a solution to our equation with $\omega=0$ and $\eta=0$ then $\varphi=$ const $\Omega$. Applying (3.10) with $\psi=\varphi$, we get

$$
\int_{B}(\delta \varphi)^{2} \mathrm{dx}=0
$$

Consequently, $\delta \varphi=0$ in $B$. Since $\varphi$ is a form of the maximal degree, our assertion follows.

Let $\pi_{h}$ and $\pi^{i}=\pi_{k}^{i}$ be the projections introduced in Section 3.1 and Section 3.3, respectively. We extend each $\pi^{i}$ and $\pi_{k}^{i}$ to the whole space $\mathfrak{H}^{p}$, by linearity, i.e.,

$$
\pi_{k}^{i}(\omega)=\omega_{k}, \quad \pi^{i}(\omega)=\sum_{k=0}^{m} \pi_{k}^{i}\left(\omega_{k}\right),
$$

if $\omega=\omega_{0}+\cdots+\omega_{m}, \omega_{k} \in \mathfrak{H}_{k}^{p}$. Put,

$$
\pi_{h}^{i}=\pi^{i} \circ \pi_{h}, \quad \pi_{h, k}^{i}=\pi_{k}^{i} \circ \pi_{h} \quad i=1, \ldots, 4 .
$$

Let $\omega$ be a restriction of a polynomial form to $\Sigma$. We define $\pi_{h}(\omega)$ by

$$
\pi_{h}(\omega)=\pi_{h}(\tilde{\omega}),
$$

if $\tilde{\omega} \in \Lambda^{p}$ and $\tilde{\omega} \mid \Sigma=\omega$. By the uniqueness of solution to the Dirichlet condition it follows easily that $\pi_{h}^{i}(\omega)$ is well defined, i.e., it does not depend on the choice of $\tilde{\omega}$. Moreover, we put

$$
\pi_{h}^{i}(\omega)=\pi^{i}\left(\pi_{h}(\omega)\right) \quad \text { and } \quad \pi_{h, k}^{i}(\omega)=\pi_{k}^{i}\left(\pi_{h}(\omega)\right) .
$$

Proposition 4.6. Let $\omega \in \mathcal{T}^{n-1}$. The differential equation $L \varphi=0$ in $B$ with

$$
(\delta \varphi)^{\mathrm{T}}=\omega \quad \text { on } \Sigma,
$$

has a solution iff $\pi_{h}^{1}(\omega)=0$. Every such solution $\varphi$ is a polynomial $n$-form. Moreover, any other solution to this equation is of the form $\varphi+\operatorname{const} \Omega$. 
Proof. Let $\tilde{\omega} \in \Lambda^{n-1}$ be such that $\omega=\pi^{\mathrm{T}}(\tilde{\omega})=\tilde{\omega}^{\mathrm{T}} \mid \Sigma$, and let $\pi_{h}(\tilde{\omega})=\tilde{\omega}_{0}+$ $\cdots+\tilde{\omega}_{m}, \tilde{\omega}_{j} \in \mathfrak{H}_{j}^{n-1}$. Then $\tilde{\omega}\left|\Sigma=\pi_{h}(\tilde{\omega})\right| \Sigma$. Thus on $\Sigma$ we have $\omega\left(\iota_{\nu} \Omega\right)=$ $\sum_{j=0}^{m} \tilde{\omega}_{j}\left(\iota_{\nu} \Omega\right)$.

By Proposition 3.1, $\chi_{n-1, j}^{0} \neq\{0\}$ iff $j=1$. Moreover, $\chi_{n-1,1}^{0}$ is spanned by $\iota_{\nu} \Omega$. Thus

$$
\pi_{h}^{1}(\tilde{\omega})=\pi_{h}^{1}\left(\tilde{\omega}_{1}\right)=C \iota_{\nu} \Omega
$$

for some real $C$. Therefore, the condition $\pi_{h}^{1}(\omega)=0$ is equivalent to $\pi^{1}\left(\tilde{\omega}_{1}\right)=0$, and consequently to $C=0$. Now by (3.3) and (3.9) one can check that

$$
\int_{\Sigma} \omega\left(\iota_{\nu} \Omega\right) \mathrm{d} \Sigma=C \int_{\Sigma}\left(\iota_{\nu} \Omega\right)^{2} \mathrm{~d} \Sigma=C \operatorname{vol} \Sigma .
$$

Suppose now that our equation has a solution. Then, by Lemma 4.5, it follows that $C=0$, and consequently $\pi_{h}^{1}(\omega)=0$.

Suppose now that $\pi_{h}^{1}(\omega)=0$. To complete the proof it suffices to indicate at least one polynomial form being a solution. Take $l=0, \ldots, m$ and let (4.5) be the decomposition of $\tilde{\omega}_{l}$. Since $\pi_{h}^{1}(\omega)=0, \alpha=0$. Let define $\zeta_{l}$ by (4.6). Since $\alpha=0$ we drop out the first component in (4.6), i.e.,

$$
\zeta_{l}=-\frac{1}{l+1} \varepsilon_{\nu} d \beta+\frac{1}{l-1} c_{\Delta}(n-1, l) \varepsilon_{\nu} d \theta .
$$

Notice that if $l=1$ then $\theta \in \chi_{n-2,0}^{0}$, so $\varepsilon_{v} d \theta$ must be zero, by Proposition 3.1. Thus,

$$
\varphi=\sum_{j=0}^{m} \zeta_{j}
$$

is a solution to our equation.

If $\omega$ is a 1 -form then $(\star \omega)\left(\iota_{\nu} \Omega\right)=\omega \nu^{\star}=\iota_{\nu} \omega$. Thus, by Proposition 4.4 and Lemma 4.5, we obtain

Lemma 4.7. Let $\omega$ be continuous normal 1-form defined on $\Sigma$. If a function $\varphi$ (smooth on the closure of $B$ ) is a solution to the equation $L \varphi=0$ in $B$ with

$$
(d \varphi)^{\mathrm{N}}=\omega \text { on } \Sigma,
$$

then

$$
\int_{\Sigma} \iota_{\nu} \omega \mathrm{d} \Sigma=0
$$

Any other solution to this equation is of the form $\varphi+$ const.

If $\omega$ is from $\mathcal{N}^{1}$, Lemma 4.7 has an algebraic interpretation analogous to that of Proposition 4.6. 
Proposition 4.8. Let $\omega \in \mathcal{N}^{1}$. The differential equation $L \varphi=0$ in $B$ with

$$
(d \varphi)^{\mathrm{N}}=\omega \quad \text { on } \Sigma,
$$

has a solution iff $\pi_{h, 1}^{4}(\omega)=0$. Every such a solution $\varphi$ is a polynomial. Any other solution to this equation is of the form $\varphi+$ const.

Proof. It suffices to show that the condition $\pi_{h, 1}^{4}(\omega)=0$ is equivalent to $\pi_{h}^{1}(\star \omega)=0$.

We know by Proposition 3.1 that $\chi_{n-1,1}^{0}$ is one-dimensional space spanned by $\iota_{\nu} \Omega$. Similarly, $I_{\Delta}(1,1) \chi_{0,0}^{0}$ is one-dimensional space spanned by $v^{\star}$, for $\chi_{0,0}^{0}=$ $\mathbb{R}$ (space of constant functions). Next, by (2.9) follows that $\pi_{h, 1}^{4}(\omega) \neq 0$ iff $\pi_{h, 1}^{1}(\star \omega) \neq 0$. Since $\chi_{n-1, k}^{0}=\{0\}$ if $k \neq 1$, our assertion follows.

\subsection{The fourth natural boundary condition $\mathcal{B}$}

Let $\mathbf{H}^{p}$ denote the space of all polynomial forms that are both closed and co-closed. Obviously, $\alpha \in \mathbf{H}^{p}$ iff $\alpha$ is a finite sum of members from $\mathbf{H}_{k}^{p}$. Let $\mathbf{H}^{p}(B)$ be the space of all forms $\alpha$ smooth on the closure of $B$ that are both closed and coclosed. Moreover, let $\sigma^{j}$ and $\pi_{h, j}^{i}$ denote the maps introduced in Section 3.3 and Section 4.2.

Lemma 4.9. Let $\omega$ and $\eta$ be a continuous tangential $(p-1)$-form and normal $(p+1)$-form defined on $\Sigma$, respectively. If a p-form $\varphi$ (smooth on the closure of $B)$ is a solution to the equation $L \varphi=0$ in $B$ with

$$
(\delta \varphi)^{\mathrm{T}}=\omega, \quad(d \varphi)^{\mathrm{N}}=\eta, \quad \text { on } \Sigma,
$$

then

$$
a \int_{\Sigma} \omega\left(\iota_{\nu} \psi\right) \mathrm{d} \Sigma=b \int_{\Sigma}\left(\iota_{\nu} \eta\right) \psi \mathrm{d} \Sigma \quad \text { for any } \psi \in \mathbf{H}^{p}(B) .
$$

Any other solution is of the form $\varphi+\alpha$ for some $\alpha \in \mathbf{H}^{p}(B)$.

Proof. On $\Sigma$ we have

$$
\begin{aligned}
(\delta \varphi)\left(\iota_{\nu} \psi\right) & =(\delta \varphi)^{\mathrm{T}}\left(\iota_{\nu} \psi\right)=\omega\left(\iota_{\nu} \psi\right), \\
\left(\iota_{\nu} d \varphi\right) \psi & =\left(\iota_{v}(d \varphi)^{\mathrm{N}}\right) \psi=\left(\iota_{\nu} \eta\right) \psi .
\end{aligned}
$$

Since $d \psi=\delta \psi=0$, applying (3.10), we obtain

$$
\begin{aligned}
0= & a\left(\int_{B}(\delta \varphi)(\delta \psi) \mathrm{dx}+\int_{\Sigma}(\delta \varphi)\left(\iota_{\nu} \psi\right) \mathrm{d} \Sigma\right) \\
& +b\left(\int_{B}(d \varphi)(d \psi) \mathrm{dx}-\int_{\Sigma}\left(\iota_{\nu} d \varphi\right) \psi \mathrm{d} \Sigma\right) \\
= & a \int_{\Sigma} \omega\left(\iota_{\nu} \psi\right) \mathrm{d} \Sigma-b \int_{\Sigma}\left(\iota_{\nu} \eta\right) \psi \mathrm{d} \Sigma .
\end{aligned}
$$


To prove the second statement of the Lemma it suffices to show that if $\varphi$ is a solution to our equation with $\omega=0$ and $\eta=0$ then $\varphi \in \mathbf{H}^{p}(B)$. Applying (3.10) we get that $\delta \varphi=0$ and $d \varphi=0$ in $B$. Thus $\varphi \in \mathbf{H}^{p}(B)$.

Let $\omega \in \mathcal{T}^{p-1}$ and $\eta \in \mathcal{N}^{p+1}$. Consider the differential equation $L \varphi=0$ in $B$ with

$$
(\delta \varphi)^{\mathrm{T}}=\omega, \quad(d \varphi)^{\mathrm{N}}=\eta \quad \text { on } \Sigma .
$$

If $p=n$ then it reduces to the equation from Proposition 4.6, if $p=0$ it reduces to the equation from Proposition 4.8. For $0<p<n$ we have

Theorem 4.10 (The fourth natural boundary condition). Let $0<p<n$, $\omega \in$ $\mathcal{T}^{p-1}$ and $\eta \in \mathcal{N}^{p+1}$. The differential equation $L \varphi=0$ in $B$ with

$$
(\delta \varphi)^{\mathrm{T}}=\omega, \quad(d \varphi)^{\mathrm{N}}=\eta \quad \text { on } \Sigma,
$$

has a solution $\varphi$ iff for any $k \geq 0$,

$$
a \pi_{h, k+1}^{1}(\omega)=b(n-p+k) \sigma^{3} \pi_{h, k+1}^{3}(\eta) .
$$

Every such a solution $\varphi$ is a polynomial form. Any other solution to this equation is of form $\varphi+\alpha$ where $\alpha \in \mathbf{H}^{p}$.

Proof. Let $\omega_{j}^{\prime}=\pi_{h, j}^{1} \omega$ and $\beta_{j}^{\prime}=\sigma^{3} \pi_{h, j}^{3} \eta$. Then $\omega_{j}^{\prime}, \beta_{j}^{\prime} \in \chi_{p-1, j}^{0}$. Fix $\psi \in \mathbf{H}_{k}^{p}$. Since $p>0$, by Corollary 3.2, it follows that $\psi=d \mu$ for some $\mu \in \chi_{p-1, k+1}^{0}$. Then by (3.3), (3.9), and the second formula of Proposition 2.1 and by Lemma 3.5 we get that the necessary condition from Lemma 4.9:

$$
a \int_{\Sigma} \omega\left(\iota_{\nu} \psi\right) \mathrm{d} \Sigma=b \int_{\Sigma}\left(\iota_{\nu} \beta\right) \psi \mathrm{d} \Sigma
$$

is equivalent to

$$
a(p+k) \int_{\Sigma} \omega_{k+1}^{\prime} \mu \mathrm{d} \Sigma=b\left(\frac{n+k-p}{n+2 k}\right) \int_{\Sigma}\left(d \beta_{k+1}^{\prime}\right)(d \mu) \mathrm{d} \Sigma .
$$

Applying (3.3) and the equality

$$
\left(d \beta_{k+1}^{\prime} \mid d \mu\right)_{p, k}=(p+k)\left(\beta_{k+1}^{\prime} \mid \mu\right)_{p-1, k+1},
$$

we get easily that

$$
a \omega_{k+1}^{\prime}=b(n-p+k) \beta_{k+1}^{\prime} .
$$

Conversely, take polynomial forms $\tilde{\omega}$ and $\tilde{\eta}$ such that $\pi^{\mathrm{T}} \tilde{\omega}=\omega$ and $\pi^{\mathrm{N}} \tilde{\eta}=\eta$ on $\Sigma$.

Suppose first that $\tilde{\omega}=\omega_{j}^{\prime}$ and $\tilde{\eta}=\varepsilon_{\nu} d \beta_{j}^{\prime}$. If $j=0$ then $\tilde{\eta}=0$. If $p>1$, $\omega_{j}^{\prime}=0$. Thus $\varphi_{0}^{\prime}=0$ is a solution to our equation. If $p=1$ then $\omega_{j}^{\prime}$ is a constant. 
We see that $\varphi_{0}^{\prime}=-(1 / n) \varepsilon_{\nu} \omega_{j}^{\prime}$ is a solution. Nevertheless, if $j=0$ then in the both cases we may write

$$
\varphi_{0}^{\prime}=\frac{1}{u_{L}(p, j+1)} I_{L}(p, j+1) \omega_{j}^{\prime} .
$$

Suppose that $j=k+1, k \geq 0$. Put $\phi=I_{L}(p, k+2) \omega_{k+1}^{\prime}$. Then by (vii) of Lemma 3.6 and the definition of $u_{L}$ we have

$$
\delta \phi=u_{L}(p, k+2) \omega_{k+1}^{\prime} .
$$

On the other hand, by (viii) of Lemma 3.6 and (4.10) we have

$$
\begin{aligned}
d \phi & =-\left(1+2 c_{L}(p, k+2)\right) \varepsilon_{v} d \omega_{k+1}^{\prime} \\
& =-\left(1+2 c_{L}(p, k+2)\right) \frac{b}{a}(n-p+k) \varepsilon_{\nu} d \beta_{k+1}^{\prime} .
\end{aligned}
$$

Now we can check easily that

$$
-\left(1+2 c_{L}(p, k+2)\right) \frac{b}{a}(n-p+k)=u_{L}(p, k+2) .
$$

Since, in our case $p<n, u_{L}(p, k+2) \neq 0$, by (3.11). Thus a polynomial form

$$
\varphi_{k+1}^{\prime}=\frac{1}{u_{L}(p, k+2)} \phi=\frac{1}{u_{L}(p, k+2)} I_{L}(p, k+2) \omega_{k+1}^{\prime}
$$

is a solution to our equation.

In the general case there exists $m \geq 0$ such that

$$
\begin{gathered}
\tilde{\omega}=\sum_{j=0}^{m}\left(\omega_{j}^{\prime}+d \omega_{j+1}^{\prime \prime}+\varepsilon_{\nu} d \alpha_{j}^{\prime}+I_{\Delta}(p-1, j) \alpha_{j-1}^{\prime \prime}\right), \\
\tilde{\eta}=\sum_{j=0}^{m}\left(\eta_{j}^{\prime}+d \eta_{j+1}^{\prime \prime}+\varepsilon_{\nu} d \beta_{j}^{\prime}+I_{\Delta}(p+1, j) \beta_{j-1}^{\prime \prime}\right)
\end{gathered}
$$

on $\Sigma$, where

$$
\omega_{j}^{\prime} \in \chi_{p-1, j}^{0}, \quad \omega_{j+1}^{\prime \prime} \in \chi_{p-2, j+1}^{0}, \quad \alpha_{j}^{\prime} \in \chi_{p-3, j}^{0}, \quad \alpha_{j}^{\prime \prime} \in \chi_{p-2, j-1}^{0},
$$

and

$$
\eta_{j}^{\prime} \in \chi_{p+1, j}^{0}, \quad \eta_{j+1}^{\prime \prime} \in \chi_{p, j+1}^{0}, \quad \beta_{j}^{\prime} \in \chi_{p-1, j}^{0}, \quad \beta_{j}^{\prime \prime} \in \chi_{p, j-1}^{0} .
$$

Since we are interested in the tangent part of $\tilde{\omega}$ and in the normal part of $\tilde{\eta}$, we may suppose that all $\alpha_{j}^{\prime}$ and $\eta_{j}^{\prime}$ are zero. Put

$$
\begin{aligned}
\varphi= & \sum_{j=0}^{m} \varphi_{j}^{\prime}+\sum_{j=0}^{m} \frac{1}{p-n-j-1} \varepsilon_{\nu} d \omega_{j+1}^{\prime \prime}+\sum_{j=0}^{m} \eta_{j+1}^{\prime \prime} \\
& +\sum_{j=0}^{m} \frac{c_{L}(p-1, j)}{n-p+j-1} \varepsilon_{\nu} d \alpha_{j-1}^{\prime \prime}+\sum_{j=0}^{m}\left(\frac{1}{p+j-1}-c_{L}(p+1, j)\right) d \beta_{j-1}^{\prime \prime} .
\end{aligned}
$$


The constants $n-p+j-1$ and $p+j-1$ we divided by, may be equal to zero iff $j=0$. But then $\alpha_{j-1}^{\prime \prime}=0$ and $\beta_{j-1}^{\prime \prime}=0$. Thus in this case the third and fourth components disappear from above sum.

One can finally check, by applying Lemma 3.6, that the polynomial form $\varphi$ is a solution to our equation.

The second part of the theorem is now a direct consequence of Lemma 4.9.

Remark. It is worth to note that the condition (4.9) from Theorem 4.10 is equivalent to the condition $\pi_{h}^{1}(\omega)=0$ from Proposition 4.6 if $p=n$, but is not equivalent to the condition $\pi_{h, 1}^{4}(\eta)=0$ if $p=0$. The reason is the shape of $\mathbf{H}_{k}^{p}$ (see Corollary 3.2). Namely, $\mathbf{H}_{k}^{n}=d \chi_{n-1, k+1}^{0}$ so for any $\psi \in \mathbf{H}_{k}^{n}$ we may repeat the reasoning of the last proof, but no form from $\mathbf{H}_{k}^{0}$ is a differential of any other form.

\section{References}

[1] I. G. AVRAMIDI, Non-Laplace type operators on manifolds with boundary, Analysis, geometry and topology of elliptic operators, World Sci. Publ., Hackensack, NJ (2006), 107140.

[2] I. G. AVRAMidi and T. P. BRANSON Heat kernel asymptotics of operators with nonLaplace principal part, Rev. Math. Phys. 13 (2001), 847-890.

[3] T. P. Branson, P. B. Gilkey and A. PierzChalski, Heat equation asymptotics of elliptic differential operators with non scalar leading symbol, Math. Nachr 166 (1994), 207-215.

[4] L. V. Ahlfors, Conditions for quasiconformal deformations in several variables, Contributions to Analysis, A collection of papers dedicated to L. Bers, Academic press, New York (1974), 19-25.

[5] L. V. AHLFORS, Invariant operators and integral representations in hyperbolic spaces, Math. Scand. 36 (1975), 27-43.

[6] L. V. AhLFors, Quasiconformal deformations and mappings in $\mathbb{R}^{n}$, J. Anal. Math. 30 (1976), 74-97.

[7] T. P. Branson, Stein-Weiss operators and ellipticity, J. Funct. Anal. 151 (1997), 334-383.

[8] T. P. BRANSON and A. PIERZCHALSKI, Natural boundary conditions for gradients, Lódź University, Faculty of Mathematics and Computer Science, preprint 2008.

[9] R. R. COIFMAN and G. WEISS, Representations of compact groups and spherical harmonics, L'Enseignement Mathematique 14 (1969), 121-175.

[10] P. B. GILKEY, "Invariance Theory, the Heat Equation, and the Atiyah-Singer Index Theorem", Publish or Perish, Wilmington, Delaware, 1984.

[11] P. B. Gilkey, T. P. Branson and S. A. Fulling, Heat equation asymptotics of "nonminimal" operators on differential forms, J. Math. Phys. 32 (1991), 2089-2091.

[12] P. B. Gilkey and L. SMITH, The eta invariant for a class of elliptic boundary value problems, Comm. Pure Appl. Math. 98 (1976), 225-240.

[13] V. P. GUSYNIN and V. V. KoRNYAK, DeWitt-Seeley-Gilkey coefficients for nonminimal operators in curved space, Fundam. Prikl. Math. 5 (1999), 649-674.

[14] Y. HommA, Bochner-Weitzenböck formulas and curvature actions on Riemannian manifolds, Trans. Amer. Math. Soc. 358 (2006), 87-114.

[15] J. Kalina, B. Ørsted, A. Pierzchalski, P. WalczaK and G. Zhang, Elliptic gradients and highest weights, Bull. Polon. Acad. Sci. Ser. Math. 44 (1996), 511-519.

[16] J. Kalina, A. Pierzchalski and P. WALCZAK, Only one of generalized gradients can be elliptic, Ann. Pol. Math. 67 (1997), 111-120. 
[17] I. KolÁŘ, P. W. MichOR and J. SLOVÁK, "Natural Operations in Differential Geometry", Springer-Verlag Berlin Heidelberg, 1993.

[18] W. KozŁows KI, Laplace type operators: Dirichlet problem, Ann. Scuola Norm. Sup. Pisa Cl. Sci. (5) 4 (2007), 53-80.

[19] A. LIPOWSKI, Boundary problems for the Ahlfors operator, (in Polish), Ph.D. Thesis, Łódź University (1996), 1-55.

[20] R. PALAIS, "Seminar on the Atiyah-Singer Index Theorem", Annals of Mathematics Studies, Vol. 57, Princeton University Press, Princeton, N.J., 1965.

[21] B. ØRSTED and A. PIERZCHALSKI, The Ahlfors Laplacian on a Riemannian manifold with boundary, Michigan Math. J. 43 (1996), 99-122.

[22] A. PIERZChAlSKI, "Geometry of Quasiconformal Deformations of Riemannian Manifolds", Łódź University Press, 1997.

[23] A. PierzChAlsKi, Ricci curvature and quasiconformal deformations of a Riemannian manifold", Manuscripta Math. 66 (1989), 113-127.

[24] H. M. ReImAnN, Rotation invariant differential equation for vector fields, Ann. Scuola Norm. Sup. Pisa Cl. Sci. 9 (1982), 160-174.

[25] H. M. Reimann, Invariant system of differential operators, Proc. of a seminar held in Torino May-June 1982, Topics in modern harmonic analysis, Instituto di Alta Matematica, Roma, 1983.

[26] E. M. STEIN and G. WeISS, Generalization of the Cauchy-Riemann equations and representation od the rotation group, Amer J. Math. 90 (1968), 163-196.

[27] E. M. SteIN and G. WeISS, "Fourier Analysis on Euclidean Spaces", Princeton University Press, 1971.

[28] H. WeYL, Das asymptotische Verteilungsgesetz der Eigenschingungen einer beliebig gestalten elastischen Koerpers, Rendiconti Cir. Mat. Palermo 39 (1915), 1-49.

Faculty of Mathematics and Computer Science Łódź University

Banacha 22, 90-238 Łódź, Poland wojciech@math.uni.lodz.pl antoni@math.uni.lodz.pl 